Article

\title{
Numerical and Experimental Efficiency Estimation in Household Battery Energy Storage Equipment
}

\author{
Matteo Moncecchi ${ }^{1, *}$, Alessandro Borselli ${ }^{1}$, Davide Falabretti ${ }^{1}$, Lorenzo Corghi ${ }^{2}$ \\ and Marco Merlo ${ }^{1, * \mathbb{D}}$ \\ 1 Politecnico di Milano, Dipartimento di Energia, Via La Masa 34, 20156 Milano, Italy; \\ alessandro.borselli@mail.polimi.it (A.B.); davide.falabretti@polimi.it (D.F.) \\ 2 UNE srl Universal Nature Energy, Via Modena 48/E, 42015 Correggio (RE); lorenzo.corghi@unesrl.com \\ * Correspondence: matteo.moncecchi@polimi.it (M.M.); marco.merlo@polimi.it (M.M.)
}

Received: 3 March 2020; Accepted: 21 May 2020; Published: 28 May 2020

\begin{abstract}
Battery energy storage systems (BESS) are spreading in several applications among transmission and distribution networks. Nevertheless, it is not straightforward to estimate their performances in real life working conditions. This work is aimed at identifying test power profiles for stationary residential storage applications capable of estimating BESS performance. The proposed approach is based on a clustering procedure devoted to group daily power profiles according to their battery efficiency. By performing a k-means clustering on a large dataset of load and generation profiles, four standard charge/discharge profiles have been identified to test BESS' performances. Different clustering approaches have been considered, each of them splitting the dataset according to different properties of the profiles. A well-performing clustering approach resulted, based on the adoption of reference parameters for the clustering process of the maximum power exchanged by the BESS and the variation of battery energy content. Firstly, the results have been proven through a numerical procedure based on a BESS electrical model and on the definition of a key performance index. Then, an experimental validation has been carried out on a pre-commercial sodium-nickel chloride BESS: this device is available in the IoT lab of Politecnico di Milano within the H2020 InteGRIDy project.
\end{abstract}

Keywords: battery energy storage systems; PV production profiles; cluster analysis; k-means algorithm; battery test profiles; sodium-nickel chloride battery; lab tests

\section{Introduction}

Electricity grids are being subject to an important transition process due to the increasing penetration of non-programmable renewable energy sources (RESs) power plants [1]. This is an important revolution in the direction of achieving the decarbonisation targets set by governments and also considering the rising awareness of environmental aspects. However, the integration of these new sources in power systems also presents some issues that need to be properly managed, such as their limited dispatchability, making the participation to the Ancillary Service Market (ASM), the lack of inertia (most of the new plants are connected to the grid by electronic power converters) problematic, and the bidirectional behaviour of the power flows on distribution networks.

Actually, the most promising solution to face these drawbacks, in a short-medium term scenario, seems to be the installation of battery energy storage systems (BESSs). These systems have assumed, in recent years, an increasing interest from both the technological and the economic point of view, also thanks to their development in other industrial sectors, such as automotive and telecommunication sectors [2]. 
Regarding power networks, BESSs are used in two different types of applications [3]. The first one is related to "front of the meter" applications, where the goal of the BESS is to support the network operation by providing services on ASM: frequency regulation, spinning reserve, and peak shaving are some examples. Another class of applications concerns "behind the meter" services, where the storage system is used by a final user to maximize his revenues (e.g., by increasing his self-consumption).

Even if BESSs can be useful in many different contexts, in the literature there is a lack of test procedures that could be used to quantify the performance of these devices in small-scale implementation.

Actually, the literature is full of contributions devoted to quantifying the performances of electrochemical cells and losses in the power conversion system; vice versa, small-scale BESS units' overall electrochemical performance evaluations are still an open issue. In particular, there is a lack of standardized testing procedures to compare different cells for a specific service: in [4] the authors assessed the performance of three different lithium batteries, adopting as a term of reference the automotive-oriented IEC 62660-1 standard [5]. This standard defines ad-hoc test conditions for lithium-ion cells in electric vehicle (EV) applications. Focusing on stationary applications, in [6] the authors developed specific testing procedures. Similarly in $[7,8]$ different performance testing protocols for lithium-ion cells were proposed. One classical approach is related to the investigation of the battery efficiency and aging under different test conditions, such as c-rate, temperature, actual operation profile, etc. Several works are proposed in this direction [9-11], but they are limited to the evaluation of the electrochemical cells.

In this paper the focus is on small-scale BESSs, suited for domestic implementation, and the goal is to evaluate the overall electrochemical performances of the equipment. In particular, in small-scale implementations, the main goal is in a reliable estimation of the BESS performances over medium/long time windows (one week, one month, one year). This is because in such devices technical data on equipment and control logics are typically limited and major uncertainties exist in the services and plant configuration that each device is asked to manage.

Taking into account that many parameters are going to impact such performance, a data-driven approach is proposed, i.e., an equivalent (empirical) model is obtained by investigating the correlation between the numerical inputs and numerical output. In particular, a procedure to define the test profiles in terms of exchanged power is proposed with the final goal to reproduce realistic in-field working conditions.

In the literature, some studies $[12,13]$ propose test profiles for frequency regulation, spinning reserve, and peak shaving. Additionally, the same authors have investigated frequency profiles to evaluate the standard performance of BESSs providing primary frequency control service [14]. However, the literature is still very poor about the self-consumption service. This lack is a real limitation for BESS development because manufacturers are not used to sharing adequate information about the real-life performance of the systems they are proposing in the market, whilst users do not have guarantees about BESS performances in performing the service required. At the present time, this uncertainty is a factor strongly impacting the market potential of these systems. For manufacturers, this issue prevents the reliable assessment of the economic value of the product sold, its actual limits, and competitiveness in the market. For users, it is very complex to evaluate the profitability of the investment, being difficult, for example, to estimate the BESS expected life or efficiency.

The present study focuses on the problem just described and aims to provide new test profiles for BESSs used for self-consumption in domestic applications. Starting from load profiles of several households, the corresponding daily battery profiles have been derived and then studied with a cluster analysis approach. In this way four clusters have been identified: profiles that belong to the same cluster have a similar impact on the battery performances. The result has been validated through numerical simulations, then it has been compared with the results of proper experimental activities conducted in the IoT lab of Politecnico di Milano-Dept. of Energy on a BESS deployed in the framework of the InteGRIDy project $[15,16]$. InteGRIDy is a H2020 project financed by the research 
and innovation programme aiming at implementing integrated smart grid cross-functional solutions for optimized synergetic energy distribution, utilization storage technologies. One of the goals of the initiative is to deploy batteries in domestic users' premises and to control them remotely to perform both front-of-the-meter and behind-the-meter services. The equipment under study is an all-in-one solution: PV MPPT, inverter, transformer, PLC, and the battery are integrated in a single cabinet [17]. In particular, a sodium-nickel chloride battery (FZSoNick 48TL200, 48 V, 200 Ah [18]) is adopted. The BESS has been made available to the IoT lab of Politecnico di Milano in order to properly design the storage control laws and to enable the remote control of the apparatus, an application of particular interest for future aggregators.

\subsection{Sodium-Nickel Chloride Battery Technology}

The literature is rich in studies related to lithium-ion technologies; it is commonly accepted that such technologies provide today the best performances. Nevertheless they are also complex (and costly) to be recycled once the end of life is reached. Therefore, in this paper the focus has been put on a different BESS technology, willing to evaluate environmentally sustainable (i.e., fully recyclable) batteries.

The electrochemical technology adopted for this study is the sodium-nickel chloride one. Operating principles of this battery technology, also known in the literature as a "ZEBRA battery", are carefully described in [19], where the authors point out an important difference with respect to conventional cells: this is a high-temperature technology, since it needs to work at about $270{ }^{\circ} \mathrm{C}$. Semi-reactions which take place at the anode and cathode during a discharging process for a cell are shown in Equations (1) and (2):

$$
\begin{gathered}
2 \mathrm{~N} \rightarrow 2 \mathrm{Na}^{+}+2 e^{-} \\
\mathrm{NiCl}_{2}+2 \mathrm{Na}^{+}+2 e^{-} \rightarrow 2 \mathrm{NaCl}+\mathrm{Ni}
\end{gathered}
$$

From a structural point of view, a cell shows a prismatic shape: in the centre there is the positive pole, consisting in a nickel current collector surrounded by other nickel metal and nickel-chloride $\left(\mathrm{NiCl}_{2}\right)$, the latter formed during charge. To complete the cathodic compartment, a secondary electrolyte $\left(\mathrm{NaAlCl}_{4}\right)$ is added in order to improve $\mathrm{Na}+$ ions' movement from the primary electrolyte towards the cathode inner region. Additionally, metal additives $\left(\mathrm{FeCl}_{2}\right)$ are put here because they can take part to the reactions and improve cell operation. All components and materials just described are enclosed by $\beta$ "-alumina, a ceramic electrolyte with a crystalline structure, that wraps the cathodic sector with a characteristic four-lobe shape on the cross-section, designed to improve the electrode-electrolyte contact area. After an interspace formed with a steel layer to exploit capillary effects, there is the anodic sector formed by sodium metal which is liquid at the typical cell operating temperature [20-22].

The main advantages of this technology concerns the intrinsic safety both during operation and in extreme conditions, recyclability of all the components, maintenance-free during life, and peak power pulse during discharge for each state of charge $(\mathrm{SoC})$ value. On the other hand, there could be important internal losses due to the need of maintaining the operating temperature, and these can overcome the advantages listed before [23]. Such a technology is nowadays considered one of the most promising for stationary applications in electrical networks [24], and so it is a valid alternative to the already well investigated lithium-ion technology. In this paper, in particular, the performance of a pre-commercial unit designed for domestic applications has been investigated. It is worthwhile to point out that the unit under test is one of the first small-size (domestic-oriented) ZEBRA BESSs so far proposed on the market.

\subsection{Battery Modeling}

Very often manufacturers' datasheets are insufficient for a proper evaluation of battery performance, so a mathematical modelling of the BESS is required. Among different types of models presented in [2], an electrical one is considered for this study since it represents the best compromise between precision and computational effort. According to the different battery technologies, the literature 
offers different solutions. For example, regarding lithium-ion, [2] describes the development of an electrical model. Regarding the ZEBRA battery [19,24] present two electrical models for the single cell, while [25] proposes an approach to electrically represent the whole battery device. Considering data availability and the detailed experimental procedure, the latter has been adopted in this study. Its equivalent electrical circuit is described in Figure 1 and consists of a voltage source connected in series with a resistor and two RC branches. The voltage source represents the open circuit voltage, the single resistor describes ohmic resistance of circuits and electrodes, while the RC branches model the transient response, respectively, due to fast and slow dynamics.

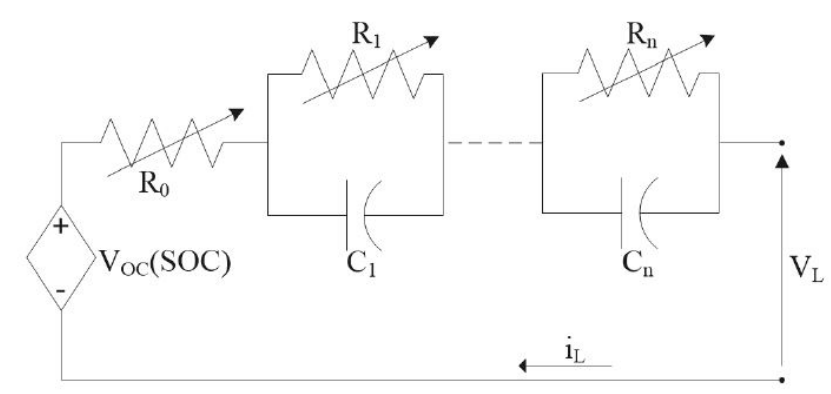

Figure 1. Equivalent electrical circuit of the battery model used in the study.

\section{Proposed Methodology}

The goal of this work is to identify a proper number of charge/discharge profiles, useful for the efficiency characterization of a BESS. To this purpose, in a first step, a set of load and generation profiles needed to be identified. In a second step, according to the power produced/absorbed locally at a given time at the user's premises, the charge/discharge profile that the BESS has to perform in order to improve the user's self-consumption is evaluated and properly clustered. In the following, the procedure adopted is described in detail.

\subsection{Load Profiles Clustering}

To the purpose of this work, a number of residential customers power profiles has been developed to test the effectiveness of a BESS in increasing the user's self-consumption. With this aim, a software called Load Profile Generator [26] has been used to produce daily load profiles of typical households with a time sample of one minute. Combining each profile with a standard PV production profile, as described in the following sections, the corresponding battery exchange power profiles is obtained. Considering that 126 different households have been developed and for each of them 365 daily profiles have been generated, the resulting dataset contains 44,115 profiles (actually, possible combinations are 45,990 , but some of them have been discarded because they were considered not relevant for the analysis, e.g., having an absence of load). It is important to underline that this software provides highly diversified profiles, considering energy saving and energy intensive households, and up to six individuals.

Remembering that one of the tasks of the work is the experimental validation of the results obtained, it has been necessary to define a procedure able to identify, among this dataset, the most characteristic profiles: these must best represent the information contained in the whole dataset. This identification process has been based on clustering algorithms.

Cluster analysis has the purpose of dividing a dataset into a certain number of groups, called clusters, so that elements of the same cluster must have similar features and, at the same time, they must also show a dissimilarity from those of another cluster. The level of similarity has been determined by algorithms that work with proper logics. One of these is k-means, which belongs to the "partitioning method" category, in which algorithms create a given number of partitions inside the initial dataset so that each element must belong to just one of these: this is the simplest and the most intuitive idea to achieve a clustering. Most of these algorithms work with an objective function based on 
Euclidean distance, which has to be properly optimzed in order to achieve similarity inside the same cluster. The objective function $E$ for k-means algorithm is reported in Equation (3), where $k$ is the number of clusters, $C_{i}$ is the $\mathrm{i}$-th cluster with the respective centroid $c_{i}$, and $p$ is the generic element of the dataset [27]. Cluster analysis works in several fields, and in the literature several studies are available $[28,29]$ in which it is applied to electric power systems, in particular for load profile analysis.

$$
E=\sum_{i=1}^{k} \sum_{p \in C i} \operatorname{dist}\left(p, c_{i}\right)^{2}
$$

\subsection{PV Production Profiles Clustering}

As already introduced, in order to define a suitable set of BESS test profiles, daily load profiles of different households have been created using the Load Profile Generator software tool. However, PV production profiles are also needed to derive battery exchange profiles. In spite this work aims at building a database of profiles representative of all possible domestic scenarios, in the direction of limiting the computational effort required, the number of production profiles to be analysed should be also limited. Therefore, the PV production side is treated with a simpler approach: cluster analysis technique is used to identify, within a set of real data, some average profiles representative of different weather and seasonal conditions. Both seasonality and weather are so dealt with a single clustering procedure. Among the average profiles identified, just the one with the highest energy content is considered, since it is the only one actually causing the BESS to charge and discharge. As a matter of fact, when PV production is insufficient, a BESS usually works only in discharging mode until the stored energy runs out. More explanations about this choice will be provided in the Section 2.3.

Several works in the literature deal with PV production profiles, such as [30-32], trying to cluster them with different approaches. In this part of the study, only partitioning methods have been taken into account in order to gain a significative result for the main concern of the work, but at the same time keeping a simple approach. In this direction, $\mathrm{k}$-means and $\mathrm{k}$-medoids algorithms have been initially considered. K-means has been firstly evaluated, even if its centroids do not belong to the initial dataset. The use of real profiles to test the BESS has been considered pivotal in this work, therefore it has been necessary to look for the dataset elements that minimise the Euclidean distance from centroids.

Before executing the algorithm, it is important to set up a proper dataset of PV daily profiles which, once clustered, can produce an output that can be coherent with the purposes of this study. Remembering that the PV production side is treated with a simpler approach considering jointly seasonal and weather variability, the results of a previous study [31] are adequate to identify some standard PV profiles analysing input all together. In fact, the authors point out that the major issues in the comparison of solar irradiance profiles are the different daylight duration and the different maximum amplitude during the year. Following the suggestion of this study, profiles have been subject to a double normalization, in power values and in the time domain, in order to cluster them considering only their shapes regardless the absolute value or daylight duration. Regarding power values, considering information availability, PV profiles have been normalized for the rated power of the respective plant. Regarding the time domain, despite the routine based on data interpolation as proposed in [31], for preserving the information content, MATLAB function resampling has been exploited to obtain profiles of 12 hours by manipulating their sampling rate.

Once the PV daily profiles dataset is set up, the clustering algorithm can be executed, but before it is important to define the number of clusters, which must be the input information of k-means. The leading idea is to limit the number of profiles and so the number of clusters for the reasons explained at the beginning of this paragraph. Considering the within-cluster sum of squares (WSS), already used in [28] for similar purposes, three clusters are adopted since WSS reduction with an additional cluster would be lower than $10 \%$. The flowchart in Figure 2 summarizes the procedure adopted. 


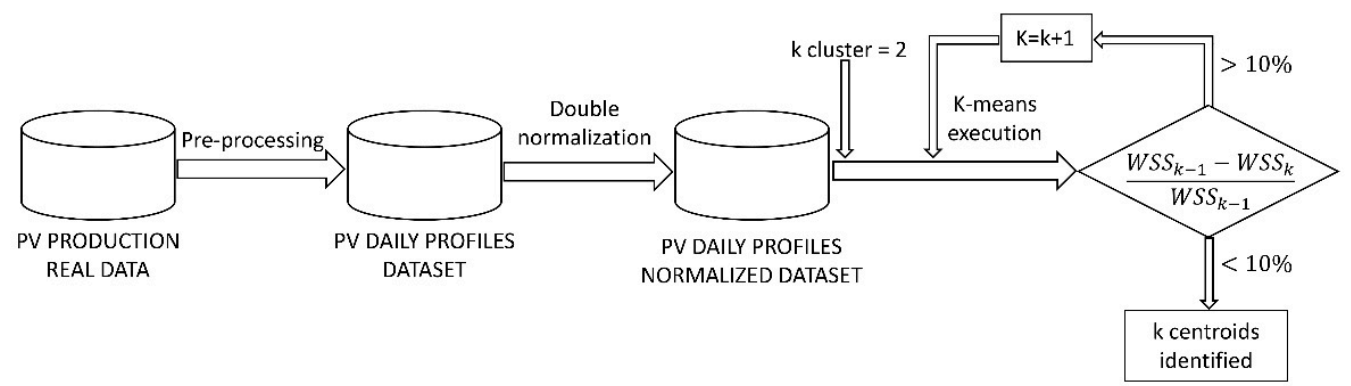

Figure 2. PV production profiles clustering flowchart of the procedure adopted.

Additionally, k-medoids have been performed using the partitioning around medoids (PAM) execution algorithm in order to preserve the consistency of its multiple-run results: this is a key aspect in the clustering technique selection and execution, as underlined in [33]. Even if this method has also been tested by the authors with the aim of exploring alternative possible approaches, the outcome confirmed the result found with $\mathrm{k}$-means, so proving the solution proposed with the procedure above.

Figure 3 shows the three normalized PV production profiles as result of this procedure, while Table 1 indicates how seasonal profiles have been distributed in the different clusters. As can be expected, it is possible to observe that the PV profile characterized by the highest energy content represents a cluster mainly composed by spring and summer elements. On the other hand, a cluster with the lowest energy content is dominated by autumn and winter profiles. It is important to underline that this PV profile clustering is controlled not only by seasonality but also by weather conditions. Considering cluster 1, profiles with a higher energy content take place in sunny days which of course are more frequent during spring and summer. However, there are also autumn and winter profiles in this cluster, and their percentage contribution is comparable with the other two seasons. Additinoally, this percentage contribution is even more comparable in cluster 2, excluding the possibility of a seasonality-driven clustering.

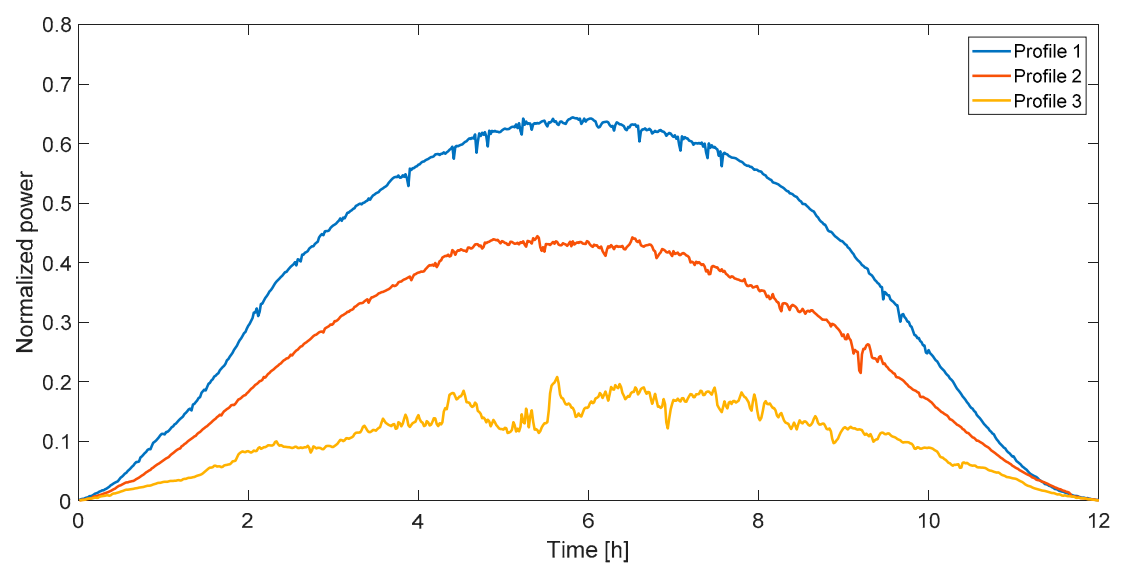

Figure 3. PV production profiles expressed in terms of normalized power and derived with the clustering procedure.

Table 1. Seasonal composition of the clusters.

\begin{tabular}{cccc}
\hline Cluster & Cluster 1 & Cluster 2 & Cluster 3 \\
\hline Spring & $26 \%$ & $16 \%$ & $4 \%$ \\
Summer & $37 \%$ & $33 \%$ & $4 \%$ \\
Autumn & $18 \%$ & $28 \%$ & $42 \%$ \\
Winter & $19 \%$ & $23 \%$ & $50 \%$ \\
& $100 \%$ & $100 \%$ & $100 \%$ \\
\hline
\end{tabular}




\subsection{Battery Profile}

The last step for the battery profile construction concerns the evaluation of the daily exchange power profile of the BESS. This is carried out by identifying the power of the BESS that, minute by minute, allows matching the load and PV production profiles (i.e., that allows zeroing the user's power exchanges with the grid). From this point of view, the power obtained by this approach can be considered an ideal power profile, which the BESS should follow during a day to maximize the self-consumption. To this purpose, the BESS exchange power profile has been determined as the algebraic sum of user load profile and PV production profile. The flowchart in Figure 4 summarizes the matching procedure adopted.

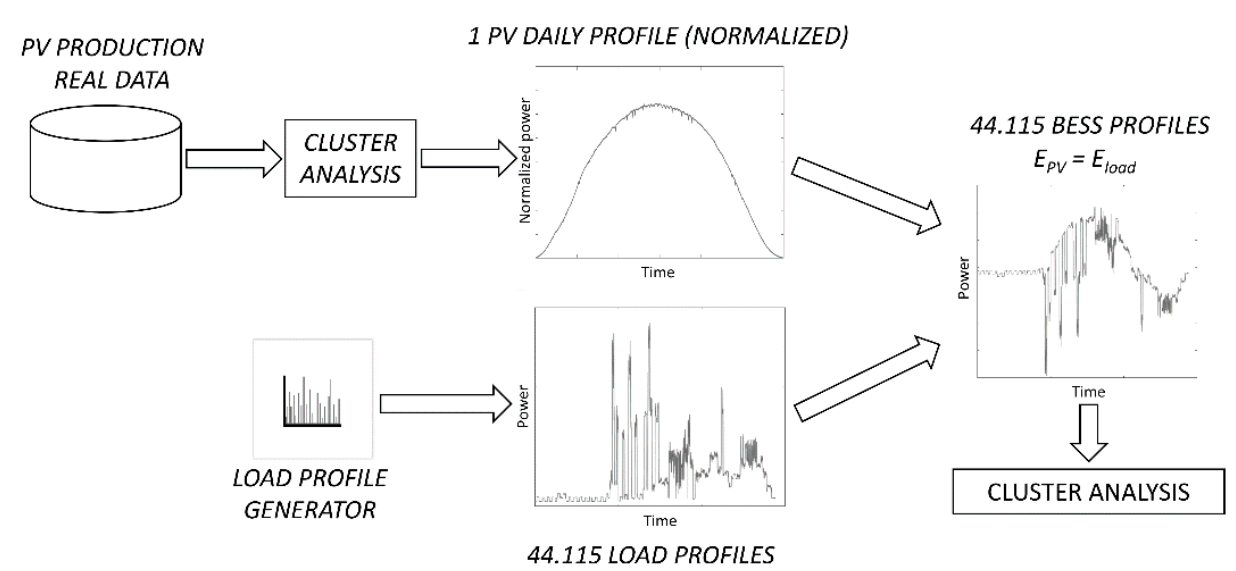

Figure 4. Battery profiles building flowchart of the procedure adopted.

It is important to point out that the proposed procedure has been subject to such a strong hypothesis in order to have a deterministic and reproducible impact of PV production on the BESS power profile. Specifically, the standard PV profile of the days with the highest energy content has been adopted as per unit reference, and the size of the PV plant for each household has been defined in order to achieve a PV production that is equivalent to the daily energy demand.

\subsection{Cluster Analysis Preliminary Considerations}

After checking that practical parameters as the number of people living in a household or the type of day (weekday, Saturday or holiday) are not good indicators to classify all the profiles, a cluster analysis was implemented by the authors to achieve the goals of the study. The flowchart in Figure 5 gives an overview of the entire procedure adopted to this purpose.

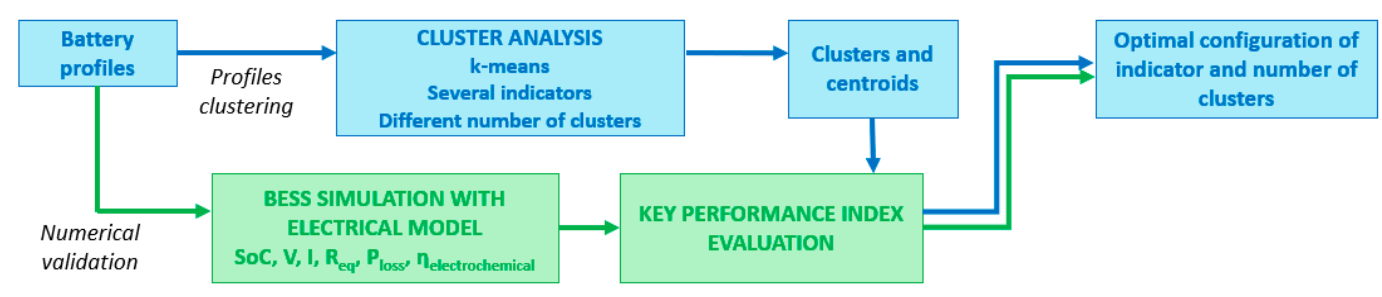

Figure 5. Battery profiles clustering and numerical validation -flowchart of the procedure adopted.

A first step concerned the choice of an appropriate clustering algorithm. Clustering algorithms can be divided in two main families: partitioning methods and density-based ones. According to [27], partitioning methods (e.g., k-means and k-medoids) evaluate Euclidean distance and look for spherically-shaped clusters, while density-based (e.g., DBSCAN and OPTICS) are suitable for evaluating clusters of various shapes. 
Given the extended field of application, the number of clustering algorithms and variants is vast. Nevertheless, for studies related to clustering profiles, the usage of $k$-means is widespread and consolidated [28,34-38]. In [28] it is used for clustering users' load profiles and authors define it as the best known and one of the most effective clustering methods. The same happens for another study [35] in which this algorithm is presented as one of the most consolidated. In another case [38], the use of k-means is justified by the ascertainment that it is the most reliable and used for load data clustering. Given these experiences and considering that the focus of the work is not the definition of an advanced algorithm, but the effective adoption of a consolidated one, k-means has been chosen for the present analysis. This algorithm is characterized by centroids as representative elements of each cluster. It is important to point out that even if a grat deal of literature studies use this algorithm, they focus on load profile clustering, and not on the battery power profile.

As asecond choice, it has been necessary to define a set of features to assume as reference to cluster the dataset. In this work, the clustering features choice has been implemented by two different approaches. Firstly, the profile description in terms of the synthetic indices reported in Figure 6 has been adopted, so performing cluster analyses on 1D, 2D, and 3D data which have been previously normalized for the maximum value of each index.

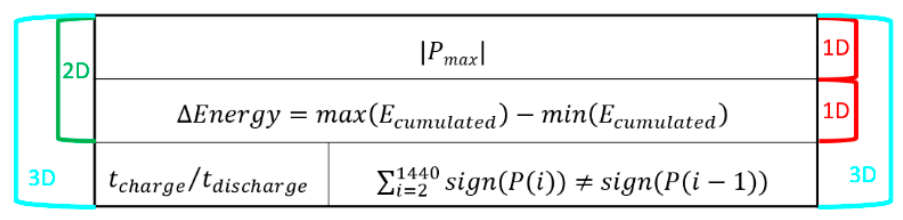

Figure 6. How synthetic indices have been grouped to define clustering features.

In Figure 6:

- $\left|P_{\max }\right|$ is the maximum power in absolute value of each daily battery profile.

- $\Delta$ Energy is the battery energy content variation, defined as the difference between maximum and minimum of the battery cumulative energy curve corresponding to each profile.

- $\quad t_{\text {charge }} / t_{\text {discharge }}$ is the time ratio between all the charge and discharge periods calculated on each daily profile.

- Number of current sign changes counts how many times the battery changes its operating condition from charge to discharge phases, and vice versa.

Secondly, other cluster analyses have been performed in order to evaluate the evolution of the profiles in time or frequency domain. In this latter case, fast Fourier transform (FFT) has been used to produce, as clustering inputs, the real and imaginary parts of the complex number representing the harmonics. In both cases, profiles have been considered as proposed in the dataset and normalized for the respective daily maximum power (in absolute value). In fact, according to [28], the use of normalized data allows focusing on the shape of each profile, regardless of physical values. On the other hand, non-normalized profiles request the analysis of both shape and physical power values, and in this way this latter aspect is favored.

The output of the clustering algorithm is the partition of the dataset in subsets, for which the overall distance between each element and the respective centroid is minimized. To obtain more robust results, the k-means clustering is repeated a given number of times using different initial centroid positions. As for Section 2.2, the only aspect that must be underlined again is that centroids identified by the algorithm do not belong to the initial dataset. In this way, remembering that one of the tasks of this work is to identify in each cluster a real profile that represents the whole group, it has been necessary to look for the dataset element that minimises the Euclidean distance from the centroid. In the following steps, these are considered as the real cluster centroids. 


\subsection{Numerical Validation}

Once obtained the clustered profiles, they needed to be numerically validated, checking if there is a real relationship between them and the BESS efficiency. In general, this assessment is not straightforward, for it is impossible to say in advance if a cluster corresponds to a specific type of user whose profiles has similar effects on the battery performance. In fact, k-means works with Euclidean distances, which may not be related to the battery efficiency corresponding to each profile.

\subsubsection{Experimental Validation}

The validation process implemented is based on a numerical procedure and an experimental assessment. The first step involves the use of the model mentioned in Section 1.2 developed in the MATLAB environment, in order to simulate each daily profile and compute the corresponding efficiency on a real BESS (the specific one used to develop the model). Then, the obtained efficiencies are compared with the clustering output. The main limit of this approach is that this comparison should be repeated for each clustering indicator in Figure 6, with the aim of understanding which of them can produce the best result. In order to compare different indicators and simultaneously evaluate the optimal number of clusters, a key performance index (KPI) has been defined.

Regarding the model simulations, three preliminary considerations are necessary. Firstly, for the electrochemical efficiency calculation, defined by Equation (4) has been adopted comparing absorbed and supplied energy over a cycle with the same initial and final SoC. Despite the assumption of changing the size of the PV plant in order to achieve equality between daily production and daily energy demand (as declared at the end of 2.3), battery energy content at the end of the day is different from that one at the beginning of the day. This is due to losses that are considered in the model. Therefore, the following procedure for SoC restoration has been defined: at the end of the profile, battery must be charged up to the initial SoC with a given power value. This value is the maximum between the power that is maintained for half of the battery charge time and $0.05 \mathrm{C}$, assuming that a so low C-rate cannot affect the performances.

$$
\eta_{\text {electr. }}=\frac{\mid \text { Energy } y_{\text {supplied }} \mid}{\mid \text { Energ } y_{\text {absorbed }} \mid} \text { with } \Delta S o C=0
$$

Secondly, considering that profiles show a characteristic timestep of one minute, an assumption has been made to approximate capacitors of the RC branches with open circuits. In this way the model has been simplified in a voltage source connected in series with three resistors. While for other BESS applications (such as electric vehicles or primary frequency control) considering the high dynamic responses of the BESS is essential, in domestic applications, since the much slower dynamics (following the daily variations of load and PV production), this aspect can be neglected in favor of a faster simulation time. The estimated error in the efficiency evaluation due to this approach is lower than $0.3 \%$, value calculated comparing the efficiency obtained simulating the BESS behavior with the complete model and the simplified one for a random extraction of 1000 profiles from the complete dataset. Considering the usual span of efficiencies for commercial BESS (in the case under analysis, for example, ranging between $90 \%$ and $100 \%$, the approximation has been considered acceptable.

Thirdly, knowing that SoC initial value has an effect on the overall efficiency, a value of $60 \%$ has been chosen as the starting point for all the simulations. Profiles that do not respect limits of battery maximum and minimum capacity have been removed.

The following step of the esperimental validation procedure is the choice of a KPI suitable for evaluating how much a clustering indicator can group profiles coherently with the battery efficiency assessed. Its definition is given in Equation (5): it evaluates the distance of profiles from the respective centroid in terms of the corresponding battery efficiency, reflecting the objective function (Equation (3)) of the k-means algorithm. Here, the Euclidean distance is replaced by a new "distance" expressed in terms of efficiencies difference. The minimum of this KPI suggests which choice, in terms of clustering 
features and number of clusters, can satisfy the final tasks, that is to define few clusters of profiles that can be easily identified with simple features, and, at the same time, can also be representative in terms of different type of usage of the battery (i.e., in term of different efficiencies).

$$
K P I=\sum_{K=1}^{n} \sum_{i=1}^{n \text { cluster }}\left(\eta_{i, K}-\eta_{\text {centroid }, K}\right)^{2}
$$

\subsubsection{Comparison with Experimental Data}

The last step of the proposed procedure provides to perform experimental tests to confirm its effectiveness in clustering the BESS power exchange profiles having similar efficiency. To this purpose, it is important to verify if the profiles identified as centroids by the method described in Section 2.5.1 show differences in terms of performance when they are tested on the pre-commercial BESS adopted in the IoT lab of Politecnico di Milano.

The experimental activity also requires some preliminary considerations. Since commercial BESSs integrate auxiliary equipment, it has been necessary to quantify their consumption through appropriate tests, for the mathematical approach proposed aims at evaluating the efficiency of the battery, not of the whole BESS. To this purpose, a definition of experimental efficiency (Equation (6)) needed to be formulated to evaluate the behaviour of the electrochemical battery unit regardless of BESS auxiliaries' consumption $E_{\text {aux }}$ :

$$
\eta_{\text {exper }}=\frac{E_{\text {load }}+E_{\text {aux }}+E_{\text {grid,injection }}}{E_{P V}+E_{\text {grid,withdrawal }}} \text { with } \Delta S o C=0
$$

In Equation (6), $E_{P V}$ is the energy measured at the PV plant's terminals. When the PV production and BESS injections are not enough, energy is absorbed from the external grid $E_{\text {grid, withdrawal }}$. This definition, for the reasons given above, considers the energy supplied to the load $E_{\text {load }}$, the one injected into the grid $E_{\text {grid,injection, }}$ and that absorbed by the auxiliaries $E_{\text {aux }}$ as useful effects. As for Equation (4), in order to obtain reliable results, efficiency must be computed on a cycle with an overall SoC variation equal to zero. Therefore, the $\mathrm{SoC}$ must be restored at the end of each profile, and to do this the same procedure described in Section 2.5.1 has been adopted.

\section{Numerical and Experimental Results}

\subsection{Numerical Validation}

Clustering has been developed according to the procedure outlined above, using the k-means algorithm to split all of the profiles in different clusters. Then, according to Section 2.5.1, a numerical validation has been performed to identify which clustering feature is the optimal one and to evaluate at the same time the number of clusters to adopt. Figure 7 presents the result of this validation, showing the KPI trend for all the features as a function of the number of clusters.

Looking at the results shown in Figure 7 and considering that the best solution is the one minimizing the value of the KPI, it can be concluded that the optimal solution is represented by the 2D clustering, based on four clusters, of profiles expressed in terms of simple indices, such as maximum power in absolute value and $\Delta$ Energy (yellow line). Contrarily to what might be expected, it emerges from the analyses that the use of simple indices always leads to a better result than more structured indicators, considering the whole profile evolution in time or frequency domain. In retrospect, it is possible to explain this considering that the maximum power managed by a battery and the maximum variation of its energy content are the quantities with the highest influence on its in-field operation, so that they are considered during the sizing phase. An interesting consideration concerns why clustering which considers the whole profile has failed (i.e., profiles classified as "similar" by this procedure actually do not present similar efficiency). A possible reason is that, considering the profile evolution, and therefore having to deal with a problem in a 1440-dimensional space, puts up several variables 
that do not influence appreciably the performance but hide the most relevant ones. The problem of clustering high-dimensional data is well known in [19], in which the authors point out that in such a case conventional distance measures can be dominated by noise. The consequence is that different approaches are required with respect to those ones used in this analysis: new algorithms must run in order to find subspaces within the data definition space, or to create a lower-dimensionality space from the original one, where clusters can be looked for properly.

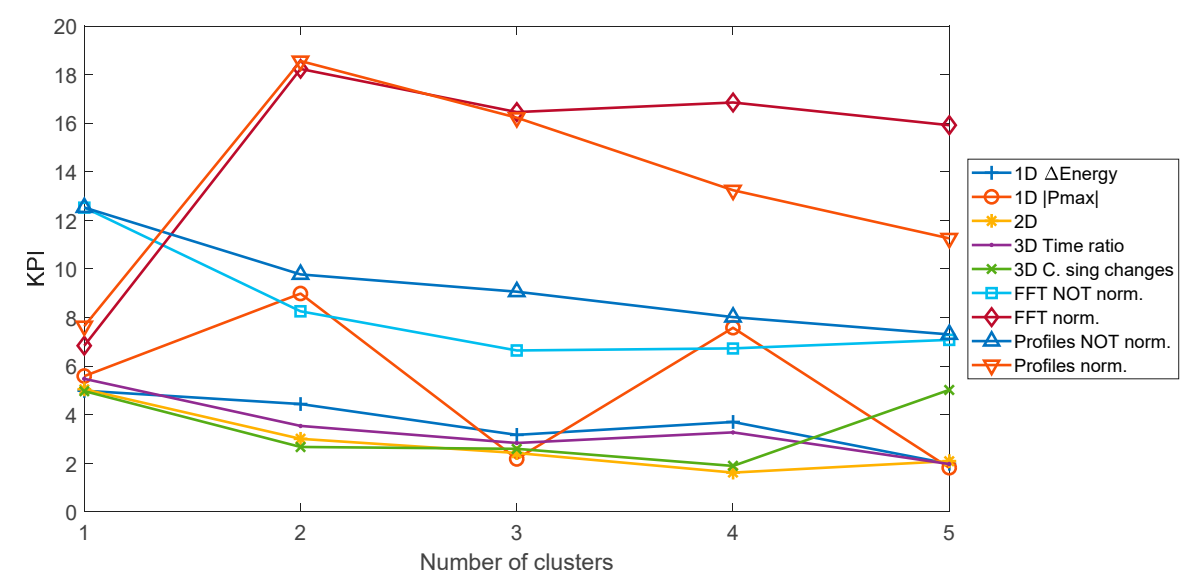

Figure 7. KPI trends as a function of clustering features and number of clusters.

Figure 8 presents the results of the numerical validation process for the optimal clustering feature, showing the electrochemical efficiency of the different profiles grouped according to the clustering output.

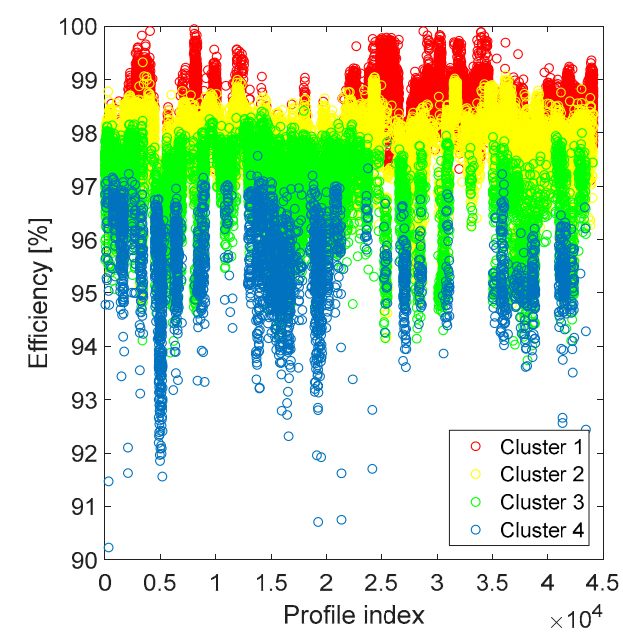

(a)

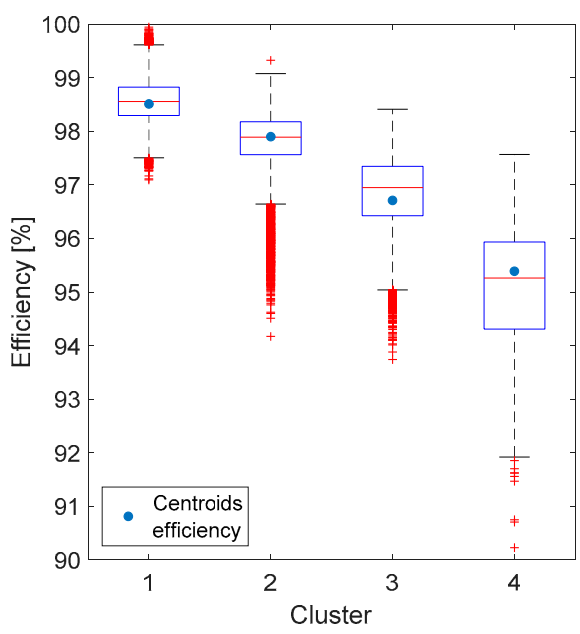

(b)

Figure 8. 2D clustering: efficiency of the different profiles grouped in clusters (a) and corresponding box plot (b).

Moreover, in Figure 9 the two-dimensional space defined by Pmax and $\Delta$ Energy is divided and coloured according to the different clusters identified. This graph can be used to classify a new battery power profile starting from these two simple indices. 


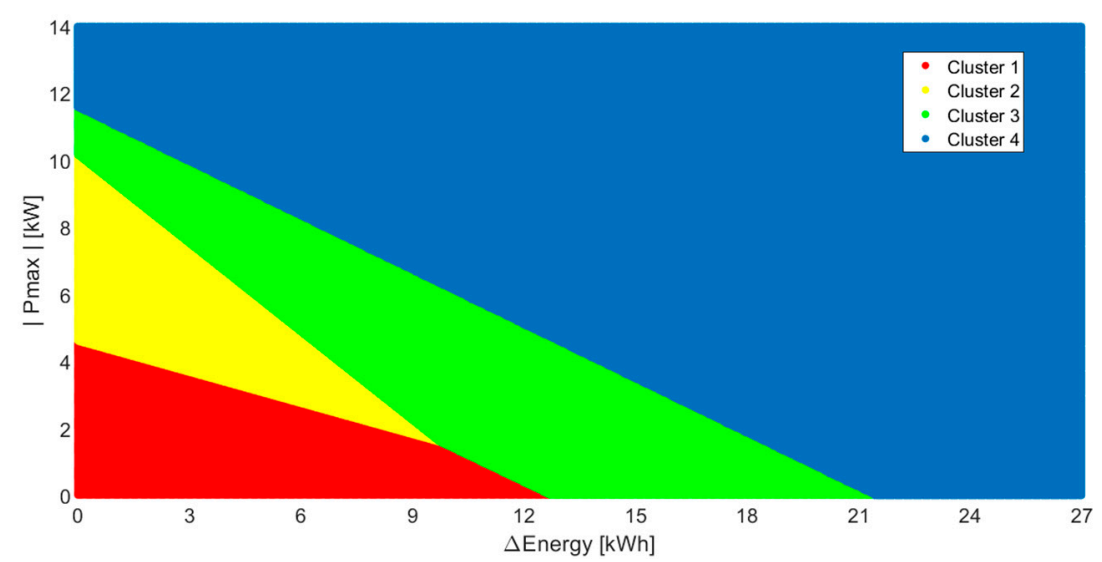

Figure 9. Regions of the Pmax and $\Delta$ Energy plan that belong to the different clusters.

Figure 10 proposes the results in time domain, showing for each cluster the centroid and the relative frequency (in terms of a chromatic scale) wherewith profiles show the different power values. In particular, the chromatic scale has been derived normalizing all the frequencies of each power value for the maximum one among them.
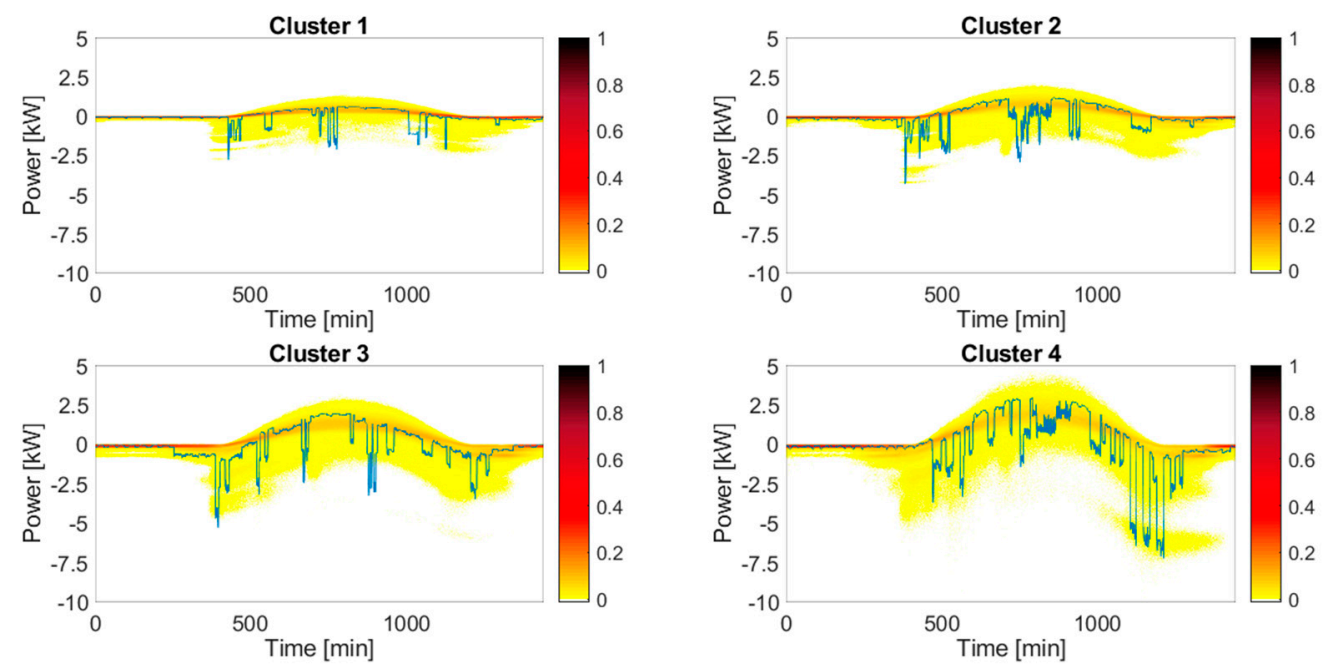

Figure 10. 2D clustering: profiles in time domain grouped by cluster and their respective centroids.

In Table 2 the main characteristics of the load profile corresponding to the centroids are described.

Table 2. Characteristics of the load profiles corresponding to the centroids.

\begin{tabular}{cccc}
\hline Centroid & $\boldsymbol{P}_{\boldsymbol{m a x}}(\mathbf{W})$ & $\boldsymbol{P}_{\boldsymbol{a v g}}(\mathbf{W})$ & $\boldsymbol{E}_{\text {daily }}(\mathbf{k W h})$ \\
\hline I & 2760 & 220 & 5.08 \\
II & 4260 & 470 & 11.29 \\
III & 5250 & 700 & 16.78 \\
IV & 7200 & 1040 & 25.04 \\
\hline
\end{tabular}

\subsection{Experimental Validation}

Then, an experimental validation has been performed. This step has required an important preliminary activity to set up a proper test bench inside the IoT lab of Politecnico di Milano. A better explanation of the whole laboratory architecture can be found in [6], while in the present work only the test bench description is reported. Figure 11 depicts the test bench showing power flows and connections for measurement and communication purposes, while Figure 12 displays the main devices. 


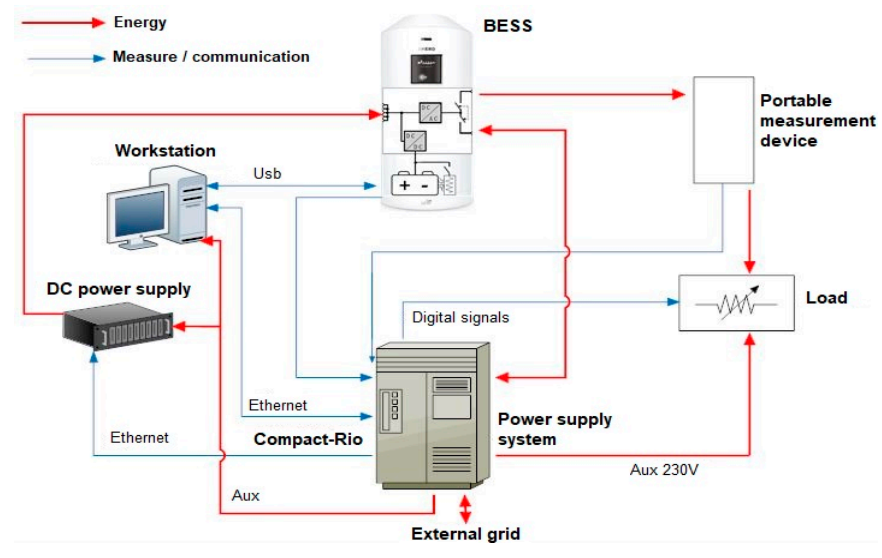

Figure 11. Test bench layout.

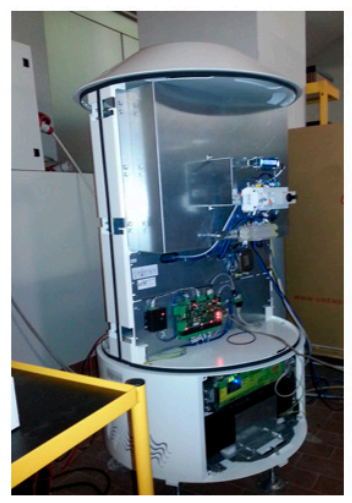

(a)

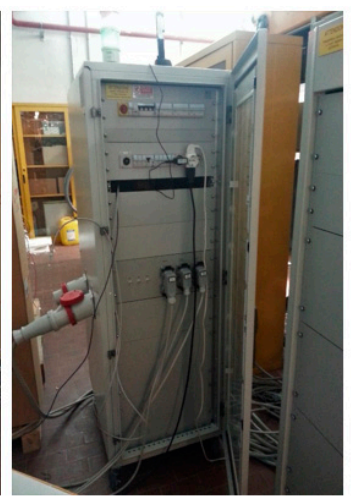

(b)

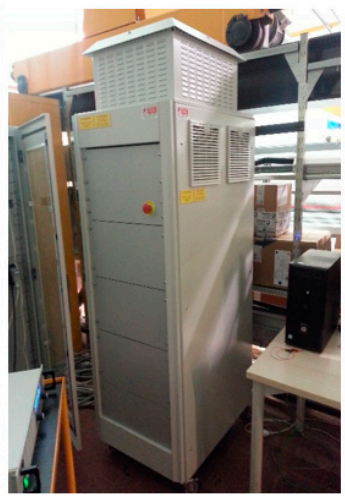

(c)

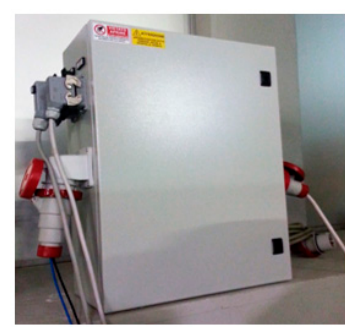

(d)

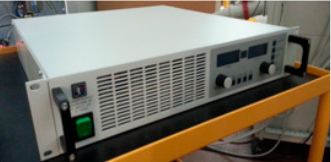

(e)

Figure 12. Devices of the test bench: (a) BESS, (b) CompactRio controller, (c) resistive load, (d) portable measurement device, (e) DC power supply.

The experimental setup is based on a CompactRio controller 9035 (National Instrument-Austin, TX, USA), a system controlled through LabView (National Instrument-Austin, TX, USA) on a workstation HP i7 (Hewlett-Packard-Palo Alto, CA, USA), which manages all the components and acquires data on them. It fulfils specific functions:

- It can modulate the power supplied by a DC power supply, produced by Elektro-Automatik (Elektro-Automatik-Viersen, Germany), model EA-PS 8360-30 2U, which emulates the production of a PV plant.

- It can modulate the power absorbed by a resistive load, realized by Ingegneria Ricerca Sistemi (IRS-Padova, Italy), which emulates the domestic load up to $5 \mathrm{~kW}$. Actually, the load is based on a resistor bank controlled by static switches; a proper code managed by LabView pilots it. 
- It measures all the electric variables exchanged by the BESS with the distribution grid and with the load, interfacing itself for this latter purpose with a portable measurement device of IRS realization. This device measures power exchanged on a single-phase line through a LEM IT 200-S Ultrastab current transducer and a LEM CV 3-1500 voltage transducer (LEM-Geneva, Switzerland).

Table 3 shows the main features resulting from the battery profiles tested on the commercial BESS, while in Table 4 the electrochemical efficiencies of the four centroids are compared with those obtained experimentally. An overall view shows that the trend defined by the cluster analysis is globally respected, therefore, the experimental tests have confirmed the theoretical outcome. However, it must be admitted that the difference between the experimental efficiencies is not so high to give a considerable margin to this comparison. On the other hand, neither electrochemical efficiencies show important differences between themselves, so, on an experimental level, with all the factors of uncertainty that may affect the test, it is reasonable that the theoretical difference has been reduced.

Table 3. Characteristics of the battery profiles tested on the commercial BESS.

\begin{tabular}{ccccc}
\hline Profile & $\boldsymbol{P}_{\text {max }, \text { charge }}(\mathbf{W})$ & $\boldsymbol{P}_{\text {max, dicharge }}(\mathbf{W})$ & $\boldsymbol{E}_{\text {delivered }}(\mathbf{k W h})$ & $\boldsymbol{E}_{\text {absorbed }}(\mathbf{k W h})$ \\
\hline I & 627 & 1386 & 3.93 & 2.80 \\
II & 802 & 2000 & 4.84 & 3.55 \\
III & 1145 & 2350 & 6.21 & 4.82 \\
IV & 1525 & 3255 & 7.89 & 5.80 \\
\hline
\end{tabular}

Table 4. Comparison between numerical and experimental electrochemical efficiency of the centroid profiles.

\begin{tabular}{ccc}
\hline Profile & Numerical Efficiency & Experimental Efficiency \\
\hline I & $98.5 \%$ & $95.4 \%$ \\
II & $97.9 \%$ & $94.9 \%$ \\
III & $96.7 \%$ & $94.5 \%$ \\
IV & $95.4 \%$ & $93.7 \%$ \\
\hline
\end{tabular}

Finally, experimental results have been also exploited to evaluate the real efficiency of the BESS, defined by Equation (7). With respect to Equation (6), which aims to identify the electrochemical efficiency of the battery, the new definition wants to evaluate the efficiency of the whole device. According to the user's point of view, it is obvious that BESS auxiliaries consumption cannot be considered as a useful effect since it actually reduces the performance of the BESS in performing the required services. Thus, the new definition differs from the previous one for the absence of the auxiliaries consumption term (now considered an internal loss of energy).

As already mentioned in Section 1.2, BESS auxiliary consumption can represent an important drawback for a given battery technology, and the extent of this drawback depends on the size of the storage and its way of use: given the storage capacity, the lower the weight of the BESS auxiliary consumption on the load daily requirement, the higher will be the efficiency of the system. Results have shown a strong efficiency reduction for those samples based on limited daily energy need. Such results are directly linked to the specific technology adopted by the electrochemical battery reporting not marginal gaps between the theoretical electrochemical efficiency and the BESS's overall one; e.g., in the scenario reported in profile IV, with a daily requirement of about $8 \mathrm{kWh}$, the auxiliary's energy need (in charge to the control of the equipment and to the regulation of the internal temperature, approx. $270^{\circ} \mathrm{C}$ ) asks for an additional $4.5 \mathrm{kWh}$; consequently the overall efficiency results limited to $60 \%$.

$$
\eta_{\text {system }}=\frac{E_{\text {load }}+E_{\text {grid,injection }}}{E_{P V}+E_{\text {grid,withdrawal }}} \text { with } \Delta S o C=0
$$




\subsection{Economic Analysis}

The economic viability of BESS investment is a factor strongly affecting the real spread of battery-related technologies in the market. Table 5 shows the data considered in input to the analysis and Table 6 presents, for the four analyzed profiles, the information regarding the number of equivalent cycles performed daily and the corresponding BESS expected life. Most of these parameters, such as the cost and price of energy and discount rate, are commonly accepted as realistic values, while the expected life has been found in technical datasheets [18]. Auxiliary's consumption has been experimentally estimated through a self-discharge test, in which equivalent cycles have been computed from experimental data according to Equation (8), considering SoC variation due to the energy absorbed and delivered during the 24 hour profile.

$$
E q_{\text {cycles }}=\frac{1}{2}\left(\frac{E_{\text {absorbed }}}{C_{\text {nom }}}+\frac{E_{\text {delivered }}}{C_{\text {nom }}}\right)
$$

Table 5. Input data for the economic analysis.

\begin{tabular}{cc}
\hline Expected life (cycles) & 3000 \\
Auxiliary consumption $(\mathrm{W})$ & 200 \\
Cost of purchased energy $(€ / \mathrm{kWh})$ & 0.16 \\
Price of injected energy $(€ / \mathrm{kWh})$ & 0.06 \\
Discount rate $(\%)$ & 4 \\
\hline
\end{tabular}

Table 6. BESS equivalent cycles and corresponding expected life.

\begin{tabular}{ccc}
\hline Profile & Equivalent Cycles & Expected Life (Years) \\
\hline I & 0.41 & 20 \\
II & 0.51 & 16 \\
III & 0.66 & 13 \\
IV & 0.84 & 10 \\
\hline
\end{tabular}

Based on these input data and on the outcome of the experimental activity, neglecting maintenance costs and battery calendar aging, operating expenses are determined by the amount of energy purchased from the grid for SoC restoration at the end of the day, while revenues deriving from PV self-consumption are measured as differential with respect to a user without BESS. Considering all this information, it is possible to quantify the discounted cash flows, whose value over the expected life is shown in Figure 13.

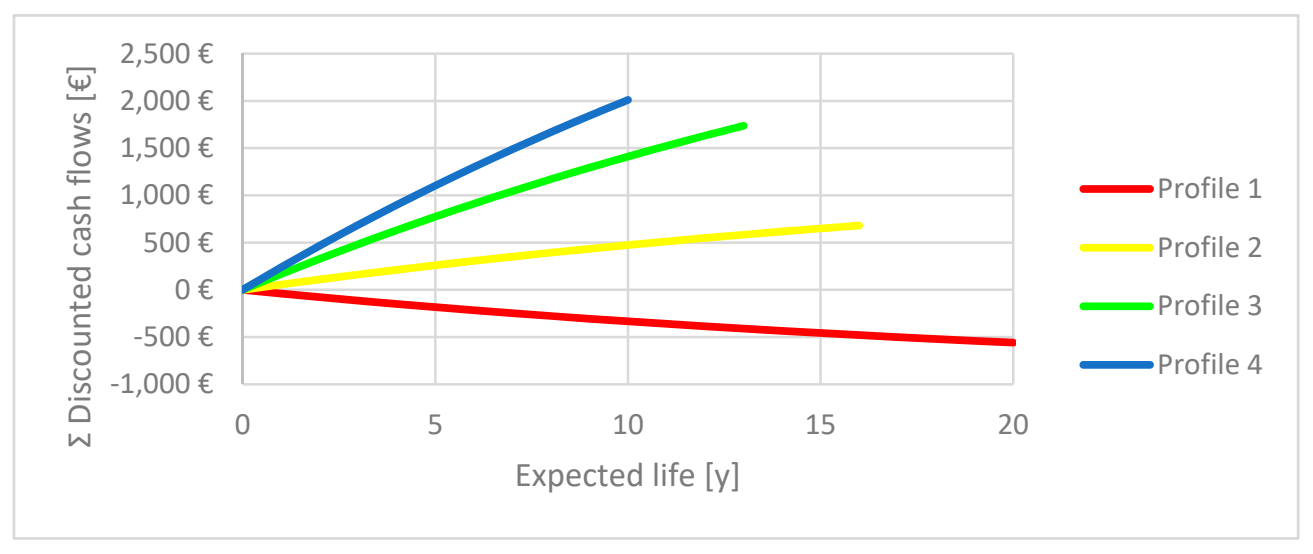

Figure 13. Discounted cash flow trends during the useful life of the BESS for the tested profiles. 
For example, assuming a profile with a daily energy of $15 \mathrm{kWh}$ and a maximum power request equal to $5 \mathrm{~kW}$, we can find from Figure 9 that the corresponding cluster is the fourth, for which we can expect a NPV around 2000 Euro and an expected life of 10 years.

This analysis clearly shows that, although the difference between the price of the energy purchased from the network and the price of the energy sold is high, energy quantities involved in residential applications are not enough to create significant profits.

\section{Conclusions and Future Developments}

The context in which this work has been developed is the key role that BESSs can cover [2], in a short-medium term scenario, to manage some issues related to the transition of electricity grids due to the increasing penetration of non-programmable RESs [1]. Despite these potentialities, the literature is still very poor in terms of profiles suitable for BESS performance evaluations in the self-consumption service. In this study, a cluster analysis on daily battery profiles has been carried out in order to identify four profiles, having a different impact on the battery performance, representative of the way of use of BESSs to perform the behind-the-meter service of self-consumption increase in a household: the greater is the requirements of the profile in terms of maximum power in absolute value and $\Delta$ Energy, the lower will be the electrochemical performance of the battery.

In this paper, an experimental validation of the approach above has been proposed. In particular, a promising technology has been investigated: a sodium-nickel chloride battery. Such a battery requires a non-marginal energy consumption for regulating the working temperature. On the other hand it is intrinsically safe, fully recyclable, and maintenance-free during life; these characteristics motivated the study regarding its applicability in a household environment.

The selected BESS technology was a perfect testbed for the proposed procedure: given the need to operate the battery at $270{ }^{\circ} \mathrm{C}$, its energetic behaviour is strongly dependent on the requested power profile. Moreover, the electrochemical efficiency does not significantly affect the overall efficiency, since the latter is strongly determined by the weight of the auxiliary device's absorption with respect to the daily requirement of the load. Actually, the sampled efficiency resulted strongly lower than the theoretical one, motivating a data-driven approach in the model (based on experimental data) rather than a detailed modelling approach; such a behavior is directly correlated with the limited power and energy capability of the domestic equipment investigated. The provided numerical results pointed out that, regardless of electrochemistry, the higher the requirement of the load the better the real performances of the device.

The tests performed demonstrated the effectiveness of the proposed procedure in classifying performances over four different clusters, identified by proper KPI. Tests are related to a control of the battery devoted to maximizing users' self-consumption (each user is provided with a suitable PV generator): given the power profile of a generic user, thanks to the proposed procedure it is possible to classify it and to estimate the BESS efficiency in providing the service.

The key aspect of this work is that three research fields (clustering and classification of PV production profiles, load profiles clustering, BESS efficiency evaluation) have been coupled to develop a new methodology aimed at obtaining a priori BESS efficiency estimation, carried out starting from the battery usage profile. Therefore, the focus of the work is more on the composition of methods resulting from the coupling and its experimental validation, rather than on the research of new approaches to perform the clustering of PV production and load profiles, or the BESS efficiency evaluation.

Load profile clustering is the research field where the authors put most effort into this work, since a very high diversification of the load profile has been achieved, as underlined in Section 2.1. The result is a double advantage with respect to other works [28,35,38], which have been, in any case, very important in terms of pointing out strengths of the k-means clustering algorithm. The first advantage is that this is one of the first works in the literature that applies a cluster analysis directly to the BESS power profiles. The second advantage is that none of these previous studies identified profiles and clusters in terms of synthetic indices, so, according to the approaches in the literature, 
a new profile allocation would require a new running of the clustering algorithm. Through this work, instead, it is possible to assign a new profile to a cluster (thanks to results in Figure 9) knowing its $\left|P_{\max }\right|$ and $\Delta$ Energy.

Author Contributions: Conceptualization: M.M. (Matteo Moncecchi) and A.B.; investigation: M.M. (Matteo Moncecchi) and A.B.; project administration: M.M. (Marco Merlo); resources: L.C.; validation: D.F. and M.M. (Marco Merlo); writing_original draft: A.B.; writing—review and editing: M.M. (Matteo Moncecchi), D.F., and M.M. (Marco Merlo). All authors have read and agreed to the published version of the manuscript.

Funding: This research was funded by European Union's Horizon 2020 research and innovation program, grant number H2020-LCE-2016-2017, LCE-02-2016, project 731268.

Conflicts of Interest: The authors declare no conflict of interest. The funders had no role in the design of the study; in the collection, analyses, or interpretation of data; in the writing of the manuscript; or in the decision to publish the results.

\section{References}

1. International Energy Agency (IEA). Electricity Information: Overview; OECD Publishing: Paris, France, 2018. [CrossRef]

2. Brivio, C.; Musolino, V.; Merlo, M.; Ballif, C. A Physically-Based Electrical Model for Lithium-Ion Cells. IEEE Trans. Energy Convers. 2019, 34, 594-603. [CrossRef]

3. Falabretti, D.; Lindholm, M.; Merlo, M.; Scapeccia, G. Energy storage coupling in a high efficiency household scenario: A real life experimental application. J. Energy Storage 2018, 17, 496-506. [CrossRef]

4. Omar, N.; Van Den Bossche, P.; Mulder, G.; Daowd, M.; Timmermans, J.M.; Van Mierlo, J.; Pauwels, S. Assessment of performance of lithium iron phosphate oxide, nickel manganese cobalt oxide and nickel cobalt aluminum oxide based cells for using in plug-in battery electric vehicle applications. In Proceedings of the 2011 IEEE Vehicle Power and Propulsion Conference, Chicago, IL, USA, 6-9 September 2011; pp. 1-7.

5. International Electrotechnical Commission IEC 62660-1: Secondary lithium-ion cells for the propulsion of electric road vehicles-Part 1: Performance testing 2018. Available online: https://webstore.iec.ch/publication/ 28965 (accessed on 26 May 2020).

6. Lazzari, R.; Micolano, E.; Conte, M.; Vellucci, F. Procedura di Prova RSE-ENEA per Batterie Litio-Ioni. 2013. Available online: https://www.enea.it/it/Ricerca_sviluppo/documenti/ricerca-di-sistema-elettrico/accumulo/ 2012/rds-2013-243.pdf (accessed on 26 May 2020).

7. Gallo, D.; Landi, C.; Luiso, M.; Rosano, A.; Landi, M.; Paciello, V. Testing protocols for battery characterization. In Proceedings of the 2014 IEEE International Instrumentation and Measurement Technology Conference (I2MTC) Proceedings, Montevideo, Uruguay, 12-15 May 2014; pp. 374-379.

8. Alessandrini, S.; Rizzuto, E.; Del Prete, Z. Characterizing different types of lithium ion cells with an automated measurement system. J. Energy Storage 2016, 7, 244-251. [CrossRef]

9. Brivio, C.; Musolino, V.; Alet, P.J.; Merlo, M.; Hutter, A.; Ballif, C. Analysis of lithium-ion cells performance, through novel test protocol for stationary applications. In Proceedings of the 20176 th International Conference on Clean Electrical Power (ICCEP), Santa Margherita Ligure, Italy, 27-29 June 2017; pp. 410-415.

10. Dubarry, M.; Qin, N.; Brooker, P. Calendar aging of commercial Li-ion cells of different chemistries-A review. Curr. Opin. Electrochem. 2018, 9, 106-113. [CrossRef]

11. Khumprom, P.; Yodo, N. Data-driven prognostic model of li-ion battery with deep learning algorithm. In Proceedings of the 2019 Annual Reliability and Maintainability Symposium (RAMS), Orlando, FL, USA, 28-31 January 2019; pp. 1-6.

12. Ferreira, S.R.; Rose, D.M.; Schoenwald, D.A.; Bray, K.; Conover, D.; Kintner-meyer, M.; Viswanathan, V. Protocol for Uniformly Measuring and Expressing the Performance of Energy Storage Systems. Off. Electr. Deliv. Energy Reliab. 2016. Available online: https://www.sandia.gov/ess-ssl/publications/SAND2016-3078R. pdf (accessed on 26 May 2020).

13. Butler, P.C.; Cole, J.F.; Taylor, P.A. Test profiles for stationary energy-storage applications. J. Power Sources 1999, 78, 176-181. [CrossRef] 
14. Falabretti, D.; Moncecchi, M.; Brivio, C.; Delfanti, M.; Merlo, M.; Musolino, V. IoT-oriented management of distributed energy storage for the primary frequency control. In Proceedings of the 2017 IEEE International Conference on Environment and Electrical Engineering and 2017 IEEE Industrial and Commercial Power Systems Europe (EEEIC / I\&CPS Europe), Milan, Italy, 6-9 June 2017.

15. InteGRIDy Project. Available online: http://www.integridy.eu/ (accessed on 26 May 2020).

16. Falabretti, D.; Moncecchi, M.; Mirbagheri, M.; Bovera, F.; Fiori, M.; Merlo, M.; Delfanti, M. San Severino Marche smart grid pilot within the InteGRIDy project. Energy Procedia 2018, 155, 431-442. [CrossRef]

17. Une, S.R.L. Zhero System. Available online: https://zherosystem.com (accessed on 26 May 2020).

18. FZSoNIck 48TL200 Battery Datasheet. Available online: https://www.fzsonick.com/applications/residentialenergy-storage (accessed on 26 May 2020).

19. Dambone Sessa, S.; Crugnola, G.; Todeschini, M.; Zin, S.; Benato, R. Sodium nickel chloride battery steady-state regime model for stationary electrical energy storage. J. Energy Storage 2016, 6, 105-115. [CrossRef]

20. Longo, S.; Antonucci, V.; Cellura, M.; Ferraro, M. Life cycle assessment of storage systems: The case study of a sodium/nickel chloride battery. J. Clean. Prod. 2014, 85, 337-346. [CrossRef]

21. Sudworth, J.L. The sodium/nickel chloride (ZEBRA) battery. J. Power Sources Elsevier 2001, 100, 149-163. [CrossRef]

22. Dustmann, C.H. Advances in ZEBRA batteries. J. Power Sources 2004, 127, 85-92. [CrossRef]

23. Bignucolo, F.; Coppo, M.; Crugnola, G.; Savio, A. Application of a simplified thermal-electric model of a sodium-nickel chloride battery energy storage system to a real case residential prosumer. Energies 2017, 10, 1497. [CrossRef]

24. Dambone Sessa, S.; Palone, F.; Necci, A.; Benato, R. Sodium-nickel chloride battery experimental transient modelling for energy stationary storage. J. Energy Storage 2017, 9, 40-46. [CrossRef]

25. Musio, M.; Damiano, A. A non-linear dynamic electrical model of Sodium-Nickel Chloride Batteries. In Proceedings of the 2015 International Conference on Renewable Energy Research and Applications (ICRERA), Palermo, Italy, 22-25 November 2015; Volume 5, pp. 872-878.

26. Load Profile Generator 7.2.0. Available online: https://www.loadprofilegenerator.de (accessed on 26 May 2020).

27. Han, J. Data Mining: Concepts and Techniques (The Morgan Kaufmann Series in Data Management Systems); Morgan Kaufmann Publishers: Waltham, MA, USA, 2011; ISBN 0123814790.

28. Azad, S.A.; Ali, A.B.M.S.; Wolfs, P. Identification of typical load profiles using K-means clustering algorithm. In Proceedings of the Asia-Pacific World Congress on Computer Science and Engineering, Nadi, Fiji, 4-5 November 2014.

29. Zufferey, T.; Ulbig, A.; Koch, S.; Hug, G. Unsupervised Learning Methods for Power System Data Analysis. In Big Data Application in Power Systems; Elsevier: Amsterdam, The Netherlands, 2018; pp. 107-124. ISBN 9780128119693.

30. Lai, C.S.; Jia, Y.; McCulloch, M.D.; Xu, Z. Daily Clearness Index Profiles Cluster Analysis for Photovoltaic System. IEEE Trans. Ind. Informatics 2017, 13, 2322-2332. [CrossRef]

31. Chicco, G.; Cocina, V.; Spertino, F. Characterization of solar irradiance profiles for photovoltaic system studies through data rescaling in time and amplitude. In Proceedings of the 2014 49th International Universities Power Engineering Conference (UPEC), Cluj-Napoca, Romania, 2-5 September 2014; pp. 1-6.

32. Muselli, M.; Poggi, P.; Notton, G.; Louche, A. Classification of typical meteorological days from global irradiation records and comparison between two Mediterranean coastal sites in Corsica Island. Energy Convers. Manag. 2000, 41, 1043-1063. [CrossRef]

33. Zhang, T.; Zhang, G.; Lu, J.; Feng, X.; Yang, W. A new index and classification approach for load pattern analysis of large electricity customers. IEEE Trans. Power Syst. 2012, 27, 153-160. [CrossRef]

34. Rhodes, J.D.; Cole, W.J.; Upshaw, C.R.; Edgar, T.F.; Webber, M.E. Clustering analysis of residential electricity demand profiles. Appl. Energy 2014, 135, 461-471. [CrossRef]

35. Pan, S.; Wang, X.; Wei, Y.; Zhang, X.; Gal, C.; Ren, G.; Yan, D.; Shi, Y.; Wu, J.; Xia, L.; et al. Cluster analysis for occupant-behavior based electricity load patterns in buildings: A case study in Shanghai residences. Build. Simul. 2017, 10, 889-898. [CrossRef] 
36. Klevas, V.; Biekša, K.; Murauskaitè, L. Innovative method of RES integration into the regional energy development scenarios. Energy Policy 2013, 64, 324-336. [CrossRef]

37. Do Carmo, C.M.R.; Christensen, T.H. Cluster analysis of residential heat load profiles and the role of technical and household characteristics. Energy Build. 2016, 125, 171-180. [CrossRef]

38. Klingler, A.-L.; Schuhmacher, F.; Wohlfarth, K. Identifying representative types of residential electricity consumers-A cluster analysis of hourly smart meter data. In Proceedings of the 4th European Conference on Behaviour and Energy Efficiency (Behave 2016), Coimbra, Portugal, 8-9 September 2016; pp. 8-9.

(C) 2020 by the authors. Licensee MDPI, Basel, Switzerland. This article is an open access article distributed under the terms and conditions of the Creative Commons Attribution (CC BY) license (http://creativecommons.org/licenses/by/4.0/). 